ARTICLE

\title{
Structural basis for specific single-stranded RNA recognition by designer pentatricopeptide repeat proteins
}

Cuicui Shen ${ }^{1, \star}$, Delin Zhang ${ }^{1, \star}$, Zeyuan Guan ${ }^{1, \star}$, Yexing Liu ${ }^{2}$, Zhao Yang ${ }^{1}$, Yan Yang ${ }^{1}$, Xiang Wang ${ }^{1}$, Qiang Wang ${ }^{1}$, QunXia Zhang ${ }^{1}$, Shilong Fan ${ }^{2}$, Tingting Zou ${ }^{1} \&$ Ping Yin ${ }^{1}$

As a large family of RNA-binding proteins, pentatricopeptide repeat (PPR) proteins mediate multiple aspects of RNA metabolism in eukaryotes. Binding to their target single-stranded RNAs (ssRNAs) in a modular and base-specific fashion, PPR proteins can serve as designable modules for gene manipulation. However, the structural basis for nucleotide-specific recognition by designer PPR (dPPR) proteins remains to be elucidated. Here, we report four crystal structures of dPPR proteins in complex with their respective ssRNA targets. The dPPR repeats are assembled into a right-handed superhelical spiral shell that embraces the ssRNA. Interactions between different PPR codes and RNA bases are observed at the atomic level, revealing the molecular basis for the modular and specific recognition patterns of the RNA bases $U, C, A$ and $G$. These structures not only provide insights into the functional study of PPR proteins but also open a path towards the potential design of synthetic sequence-specific RNA-binding proteins.

\footnotetext{
${ }^{1}$ National Key Laboratory of Crop Genetic Improvement and National Centre of Plant Gene Research, Huazhong Agricultural University, Wuhan 430070, China. ${ }^{2}$ Center for Structural Biology, School of Life Science, Tsinghua University, Beijing 100084, China. ${ }^{\star}$ These authors contributed equally to this work. Correspondence and requests for materials should be addressed to P.Y. (email: yinping@mail.hzau.edu.cn).
} 
$\mathrm{T}$ he ability to design proteins to manipulate specified target DNA/RNA sequences has been a long-sought but elusive goal ${ }^{1}$. The modular mode of target recognition by specific proteins allows the development of DNA/RNA-binding tools through the assembly of particular motifs or domains ${ }^{2}$. Despite encouraging progress in the realm of DNA editing ${ }^{1}$, including, for example, the successful application of zinc finger domains ${ }^{3}$, transcription activator-like effectors (TALE) ${ }^{4,5}$ and the clustered regularly interspaced short palindromic repeat (CRISPR)-Cas9 system $^{6,7}$ in targeted gene regulation, our knowledge of how to design proteins that can selectively bind desired RNA sequences remains limited. Pumilio and FBF homology (PUF) family proteins contain an RNA-binding domain that characteristically comprises eight $\alpha$-helical repeats, each of which recognizes one RNA base ${ }^{8}$. The application of the PUF domain involves eight/ sixteen repeats, each binding a specific nucleotide, to generate RNA-recognition tools ${ }^{9,10}$. Nevertheless, there are limits to the further application of engineered PUF domains ${ }^{2}$. Thus, more candidates with a potential for RNA manipulation are required to enrich the gene-regulation toolbox. Containing a tandem array of 2-30 repeats, pentatricopeptide repeat (PPR) proteins constitute one of the largest protein families in land plants ${ }^{11,12}$. Each PPR repeat aligns to a single nucleotide of the RNA target in modular patterns, making these proteins suitable for the development of exciting new biotechnologies ${ }^{13-16}$.

A typical PPR repeat, also referred to as a PPR motif ${ }^{17}$, is defined as a degenerate 35-amino-acid repeat (hence the term pentatricopeptide) that folds into a hairpin of antiparallel alpha helices, as revealed by the crystal structures of the PPR domains of protein-only RNase P1(ref. 18) from Arabidopsis thaliana and of human mitochondrial RNA polymerase ${ }^{19,20}$, and by the structures of the RNA-bound PPR repeat assembly of PPR $10^{21,22}$ and Thylakoid assembly 8 (THA 8$)^{23,24}$. Within each repeat, the combinations of two amino acids, known as the PPR code at two key positions (the 5th and the 35th) confer RNA specificity ${ }^{15,25,26}$. The structure of the PSAJ-PPR10 complex corroborates this binary code model ${ }^{22}$, demonstrating that these two amino acids interact directly with the target nucleobases. More than 30 combinations of PPR code amino acids, such as 'ND (asparagine and aspartate)' and 'TD (threonine and aspartate)', have been predicted by bioinformatic studies ${ }^{25-27}$. However, the specific nucleotide targets binding to them have been only partially identified by biochemical and structural studies ${ }^{21,22,28-30}$. Until now, the PPR code remains largely enigmatic, requiring further elucidation ${ }^{15,26}$.

In the past decade, many PPR genes have been identified and extensively studied ${ }^{15,26}$. PPR proteins function in various aspects of RNA metabolism, primarily in organelles, facilitating the editing $^{31}$, processing ${ }^{32}$, splicing ${ }^{33}$ and translation of RNAs ${ }^{34}$. However, most of their functions remain unclear because their target RNA sequences are incompletely understood. Deciphering the PPR code will greatly facilitate precise RNA target prediction and identification and the functional investigation of PPR proteins, which requires elaborate structural information about the PPR-RNA complex ${ }^{15}$.

Previously, our group has successfully designed 35-amino-acid PPR repeat scaffolds, determined via comprehensive computational homology analysis of P-type PPR proteins from A. thalian ${ }^{28}$. We assembled PPR repeat scaffolds in tandem and fused parts of PPR10 from Zea mays onto the amino and carboxyl termini. Engineered designer (dPPR) proteins can specifically select their predicted RNA targets according to PPR codes in vitro. Artificial manipulation of the PPR code amino acids, rather than the PPR repeat scaffolds, leads to specific RNA recognition. Similar results and crystal structures of artificially engineered PPR proteins free of target RNA have also been reported by other groups ${ }^{29,35}$. These results suggest that PPR scaffolds are amenable to the engineering of designer RNAbinding domains, as promising tools to achieve specific RNA recognition in vitro. Despite these advances, the lack of a structure of a dPPR-RNA complex has hindered the elucidation of RNA recognition by dPPR proteins and, more importantly, has restricted the development of more efficient RNA manipulation tools.

To determine how dPPR proteins specifically accommodate and recognize their single-stranded RNA (ssRNA) targets, we designed and purified a set of homogeneous dPPR proteins with high-target recognition specificity and solved the crystal structures of four different dPPR proteins in complex with their respective target ssRNAs. A typical RNA-bound dPPR protein adopts a right-handed superhelical spiral structure, with its target ssRNA molecule sitting in the interior cavity and interacting with corresponding PPR repeats in a modular fashion. These structures also reveal atomic-level interaction patterns among all four RNA bases (U, C, A and G) with different PPR codes. On the basis of our structural discoveries, models of RNA recognition by some additional PPR codes were verified. Together, our findings not only provide detailed modular and specific binding patterns of dPPR repeat, but also establish an important framework for gene-manipulation applications.

\section{Results}

RNA sequence selectivity of dPPR proteins. On the basis of previous investigation ${ }^{28}$, we used dPPR scaffolds with four different codes: ND, NS, SN and TD (Fig. 1a). Each of the dPPR repeat scaffolds contains 35 amino acids, and those at positions 5 and 35 are referred to as PPR code amino acids. Parts of PPR10 from Z. mays were fused onto the amino and carboxyl termini of a series of tandem dPPR repeats as amino-terminal domain (NTD) and carboxyl-terminal domain (CTD), to enhance the solubility of the engineered protein (Fig. $1 \mathrm{~b}$ and Supplementary Fig. 1). To verify the RNA selectivity, the dPPR proteins dPPR$\mathrm{U}_{8} \mathrm{~N}_{2}$ (in which $\mathrm{N}$ indicates any nucleotide) comprising $10 \mathrm{dPPR}$ repeats with different 5th and 6th repeats with different PPR codes were constructed and purified to homogeneity. Each of the four dPPR $-\mathrm{U}_{8} \mathrm{~N}_{2}$ proteins specifically bound to its respective target ssRNA with a dissociation constant of $\sim 20-75 \mathrm{nM}$, as estimated on the basis of the results of electrophoretic mobility shift assay (EMSA) (Fig. 1c; Supplementary Fig. 2 and Supplementary Table 1). The substitution of any target RNA base with another led to a notable reduction in or complete abrogation of dPPR- $\mathrm{U}_{8} \mathrm{~N}_{2}$ binding (Fig. 1c and Supplementary Fig. 2). For instance, the substitution of cytosine at positions 5 and 6 with the pyrimidine uracil or the purines adenine and guanine resulted in the dissociation of the dPPR-RNA complex.

Furthermore, we were able to target a specific ssRNA sequence by assembling $\mathrm{APPR}$ proteins with a combination of four types of PPR codes. For example, dPPR-4, which comprises eight dPPR repeats with all four types of dPPR code, bound to its predicted target RNA with high specificity as expected. The dissociation constant was $\sim 25 \mathrm{nM}$ (Supplementary Fig. 3 and Supplementary Table 1). In summary, dPPR proteins can distinguish target sequences with high specificity.

Crystallization of RNA-bound dPPR proteins. To elucidate the atomic mechanism of ssRNA recognition by dPPR proteins, we launched a systematic effort to obtain the crystal structure of functional dPPR in complex with target RNA. On the basis of the hypothesis that the number of $\mathrm{dPPR}$ repeats might influence crystallization by affecting molecular packing, we altered the number of $\mathrm{dPPR}$ repeats and purified many batches of $\mathrm{dPPR}$ 
a

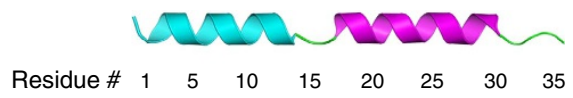

Repeat-U VVTYNTLIDGLCKAGKLDEALKLFEEMVEKGIKPD

Repeat-C VVTYNTLIDGLCKAGKLDEALKLFEEMVEKGIKPS

Repeat-A VVTYSTLIDGLCKAGKLDEALKLFEEMVEKGIKPN

Repeat-G VVTYTTLIDGLCKAGKLDEALKLFEEMVEKGIKPD

\section{b}

dPPR- $U_{10}$

Target RNA

NTD NDNDNDNDNDNDNDNDNDNDCTD

$5^{\prime}-\cup \cup \cup \cup \cup \cup \cup \cup \cup \cup-3^{\prime}$

$\mathrm{dPPR}-\mathrm{U}_{8} \mathrm{C}_{2}$ NTD NDNDNDNDNS NS NDNDNDNDCTD

Target RNA2 $5^{\prime}-U \cup \cup \cup C C \cup \cup \cup U$ - $U$ '

dPPR- $\mathrm{U}_{8} \mathrm{~A}_{2}$ NTD NDNDNDND SN SN NDNDNDNDCTD

Target RNA3 $5^{\prime}-U \cup \cup \cup A A \cup \cup \cup \cup-3^{\prime}$

dPPR $-\mathrm{U}_{8} \mathrm{G}_{2}$ NTD NDNDNDNDTD TD NDNDNDNDCTD

Target RNA4

C

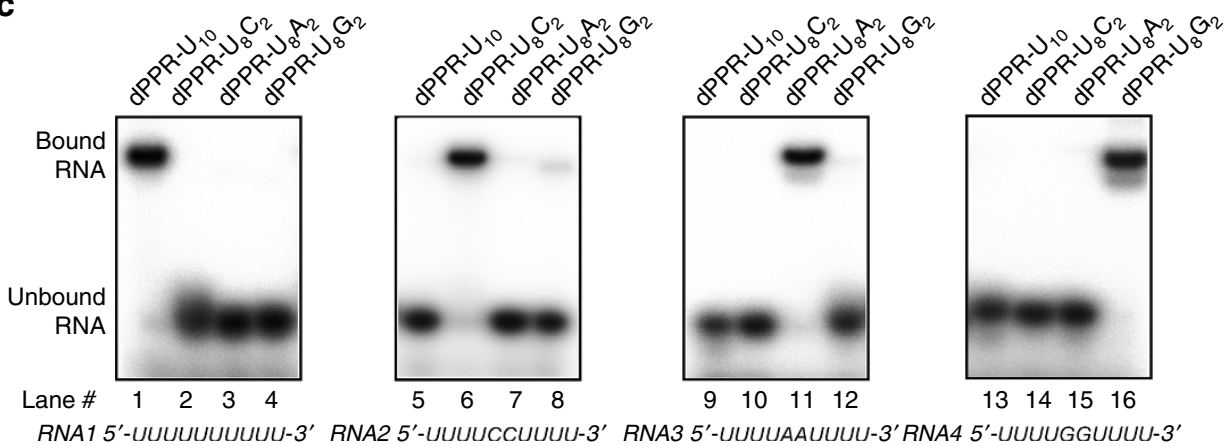

Figure 1 | Design of dPPR proteins with RNA-recognition specificity. (a) Sequence of dPPR motif containing 35 amino acids. The secondary structural elements of a typical PPR motif are shown above. PPR codes comprising two residues located at the 5th and 35th positions are labelled in distinct colours ('ND', 'NS', 'SN' and 'TD', which recognize uracil, cytosine, adenine and guanine, respectively, are coloured green, lilac, yellow and cyan, respectively). Single-letter abbreviations for the amino acid residues are as follows: A, Ala; C, Cys; D, Asp; E, Glu; F, Phe; G, Gly; I, lle; K, Lys; L, Leu; M, Met; N, Asn; P, Pro; $\mathrm{S}$, Ser; T, Thr; $V$, Val and $Y$, Tyr. (b) Schematic representation of dPPR- $U_{10}$, dPPR $-U_{8} C_{2}, d P P R-U_{8} A_{2}$ and $d P P R-U_{8} G_{2}$, and their targeting of specific RNA sequences. The shaded binary amino acids indicate PPR repeats with different codes. The NTD and CTD are from native PPR10. (c) Specific RNA target binding of dPPRs. In the RNA EMSA, $50 \mathrm{nM}$ purified dPPRs were mixed with $2 \mathrm{nM}{ }^{32} \mathrm{P}$-labelled RNA, respectively. The sequence of the RNA probe is listed below each panel. Fig. 1a is reprinted from, Shen et al. ${ }^{28}$ with permission from Elsevier.

\begin{tabular}{|c|c|c|c|c|}
\hline & dPPR-U $\mathbf{U}_{10}$ & dPPR $-U_{8} C_{2}$ & dPPR- $\mathbf{U}_{8} \mathbf{A}_{2}$ & $d P P R-U_{8} G_{2}$ \\
\hline \multicolumn{5}{|l|}{ Data collection } \\
\hline Space group & $\mathrm{P} 22_{1} 2_{1} 2_{1}$ & $\mathrm{P} 22_{1} 2_{1} 2_{1}$ & $\mathrm{P} 22_{1} 2_{1} 2_{1}$ & $\mathrm{P} 22_{1} 2_{1} 2_{1}$ \\
\hline \multicolumn{5}{|l|}{ Cell dimensions } \\
\hline$a, b, c(\AA)$ & $52.73,85.27,95.10$ & $51.43,84.78,94.36$ & $52.55,84.90,95.90$ & $52.06,85.10,95.40$ \\
\hline$\alpha, \beta, \gamma\left(^{\circ}\right)$ & $90.00,90.00,90.00$ & $90.00,90.00,90.00$ & $90.00,90.00,90.00$ & $90.00,90.00,90.00$ \\
\hline Resolution $(\AA)$ & $40-2.20(2.28-2.20)$ & $40-2.30(2.38-2.30)$ & $47.7-2.60(2.71-2.60)$ & $47.7-2.50(2.61-2.50)$ \\
\hline$R_{\text {merge }}(\%)$ & $6.8(56.8)$ & $7.2(20.8)$ & $10.0(46.5)$ & $8.2(5.4)$ \\
\hline $1 / \sigma$ & $21.1(2.6)$ & $26.3(3.2)$ & $16.7(5.5)$ & $19.8(4.9)$ \\
\hline Completeness (\%) & $99.0(99.0)$ & $98.4(87.9)$ & $99.8(98.3)$ & $99.8(98.5)$ \\
\hline Redundancy & $3.7(3.4)$ & $6.0(5.8)$ & $8.6(10.9)$ & $10.1(12.8)$ \\
\hline \multicolumn{5}{|l|}{ Refinement } \\
\hline Resolution $(\AA)$ & $38.90-2.19$ & $38.67-2.29$ & $46.08-2.60$ & $47.70-2.50$ \\
\hline No. reflections & 22,260 & 18,753 & 13,769 & 15,113 \\
\hline$R_{\text {work }} / R_{\text {free }}(\%)$ & $25.03 / 29.94$ & $22.51 / 24.75$ & $26.69 / 29.30$ & $22.30 / 29.60$ \\
\hline \multicolumn{5}{|l|}{ No. atoms } \\
\hline Protein & 2,990 & 2,860 & 2,778 & 2,850 \\
\hline Ligand/ion & 204 & 208 & 198 & 225 \\
\hline Water & 76 & 67 & 41 & 49 \\
\hline \multicolumn{5}{|l|}{ B-factors } \\
\hline Protein & 56.4 & 53.3 & 52.0 & 57.1 \\
\hline Ligand/ion & 44.2 & 49.3 & 41.3 & 49.5 \\
\hline Water & 54.1 & 52.6 & 47.2 & 46.8 \\
\hline \multicolumn{5}{|l|}{ R.m.s. deviations } \\
\hline Bond lengths $(\AA)$ & 0.010 & 0.016 & 0.015 & 0.007 \\
\hline Bond angles $\left({ }^{\circ}\right)$ & 1.241 & 1.802 & 1.793 & 0.917 \\
\hline
\end{tabular}

R.m.s., root mean square.

Values in parentheses are for the highest resolution shell. $R_{\text {merge }}=\Sigma_{h} \Sigma_{i}\left|I_{h, i}-I_{h}\right| / \Sigma_{h} \Sigma_{i} h_{h, i}$ where $I_{h}$ is the mean intensity of the $i$ observations of symmetry related reflections of $h . R=\Sigma\left|F_{\text {obs }}-F_{\text {calc }}\right| / \Sigma F_{\text {obs }}$ where $F_{\text {calc }}$ is the calculated protein structure factor from the atomic model $\left(R_{\text {free }}\right.$ was calculated with $5 \%$ of the reflections selected). 
proteins with different repeat numbers for crystallization. After numerous unsuccessful trials, we finally succeeded in crystallizing each dPPR- $\mathrm{U}_{8} \mathrm{~N}_{2}$ in complex with its respective target ssRNA $\left(5^{\prime}\right.$-UUUUNNUUUU-3'), in space group $\mathrm{P} 2{ }_{1} 2_{1} 2_{1}$ (Methods). These four structures were determined by molecular replacement (MR) using the atomic coordinates of the consensus $\mathrm{PPR}^{29}$ (cPPR, PDB accession code 4PJR), and were refined to resolutions of $2.20,2.30,2.60$ and $2.53 \AA$, for dPPR- $\mathrm{U}_{10}, \mathrm{dPPR}-\mathrm{U}_{8} \mathrm{C}_{2}$, dPPR$\mathrm{U}_{8} \mathrm{~A}_{2}$ and $\mathrm{dPPR}-\mathrm{U}_{8} \mathrm{G}_{2}$, respectively (Table 1 ). When $\mathrm{dPPR}-\mathrm{U}_{8} \mathrm{C}_{2}$ was superimposed on dPPR- $\mathrm{U}_{10}$, dPPR- $\mathrm{U}_{8} \mathrm{~A}_{2}$ and $\mathrm{dPPR}-\mathrm{U}_{8} \mathrm{G}_{2}$, the root mean square deviations were $0.73,0.99$ and $1.00 \AA$ over 348,367 and $374 \mathrm{C} \alpha$ atoms, respectively (Supplementary Fig. $4 \mathrm{~b}$ ). Because the four structures exhibited almost identical features except for the RNA base binding details of repeats 5 and 6 , we focused on describing the structure of RNA-bound $d P P R-U_{8} C_{2}$.

RNA-bound dPPR adopts a right-handed superhelical structure. In the complex structure, $\mathrm{dPPR}-\mathrm{U}_{8} \mathrm{C}_{2}$ has $10 \mathrm{dPPR}$ repeats (residues 174-523), which are capped by NTD and CTD helices (Fig. 2a). Each repeat in $\mathrm{dPPR}-\mathrm{U}_{8} \mathrm{C}_{2}$ contains 35 amino acids, forming a hairpin of $\alpha$-helices that both contain four helical turns followed by a five-residue loop. The two helices, formed by residues 1-14 and 17-30 (Fig. 1a), are designated as helix a and helix b, respectively (Fig. 2a, left panel). The whole-protein molecule has an overall appearance of a solenoid with a polar axis of $75 \AA$ and a diameter of $50 \AA$ (Fig. 2a). The internal layer along the superhelical axis is constituted by helices a, whereas helices b outline the external layer of the superhelix. Following the assignment of dPPR proteins in the electron density maps, electron densities indicative of RNA bases, which interdigitated with PPR helices, emerged in
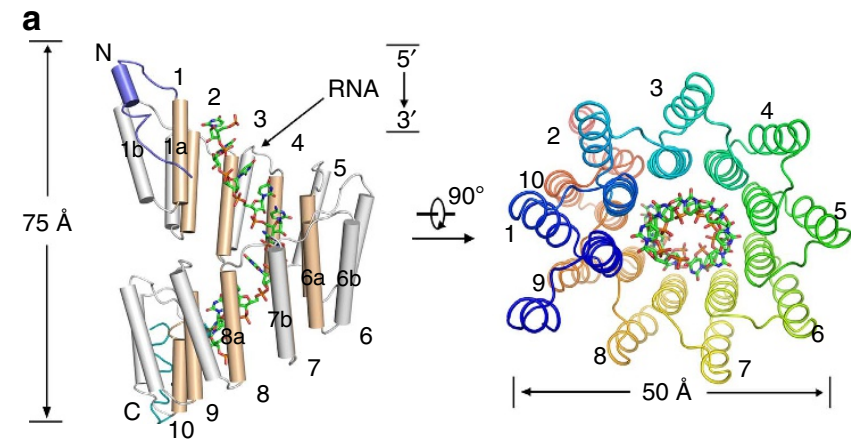

b
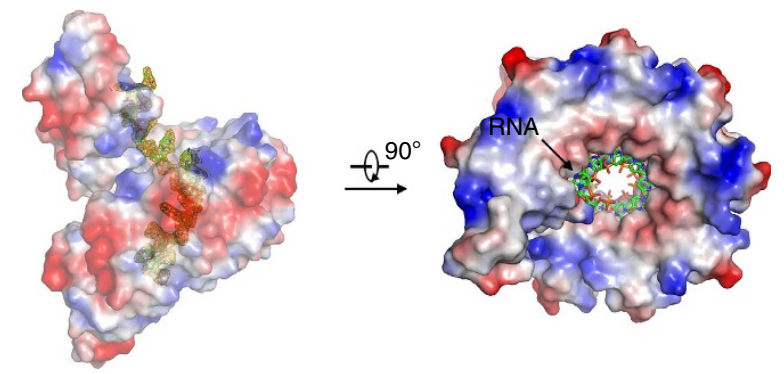

Figure 2 | Overall structure of RNA-bound $\mathbf{d P P R}-\mathbf{U}_{\mathbf{8}} \mathbf{C}_{\mathbf{2}}$. (a) Overall structure of dPPR $-U_{8} C_{2}$ bound to its target RNA element. $d P P R-U_{8} C_{2}$ comprises 10 repeats capped by a small NTD helix (slate) and a CTD helix (cyan). The $10 \mathrm{dPPR}$ repeats of $\mathrm{dPPR}-\mathrm{U}_{8} \mathrm{C}_{2}$ form a right-handed superhelical assembly. Wheat and grey bundles indicate helix $a$ and helix $b$, respectively. (b) Electron density of target RNA is clearly visible in the cavity of the dPPR superhelix. The electron density, contoured at $1 \sigma$, is shown in yellow. The surface electrostatic potential was calculated with PyMOL. Two perpendicular views are presented, with the ssRNA molecule depicted as sticks. All structure figures were prepared using PyMOL. the cavity of the superhelix (Fig. 2b and Supplementary Fig. 4c). Because of the limited quality of the electron density data, only the 10 nucleotides coordinated by repeats could be modelled. Only one complex comprising one dPPR molecule with an ssRNA target was present in each asymmetric unit, similarly to the solution complex structure of $A T P H$-bound PPR $10^{30}$. The overall dPPR protein structure consists of repetitions of helix pairs packing against each other to form a right-handed superhelical spiral shell that embraces its target ssRNA. The ssRNA molecule forms a right-handed parallel duplex structure with an 'outer-layer' spiral protein enclosure. All 10 nucleotides in the target RNA elements strictly exhibit the modular pattern binding to corresponding $\mathrm{dPPR}$ repeats.

Structural explanation of conformational plasticity of dPPR. Similarly to RNA-free PPR10, all dPPR $-\mathrm{U}_{8} \mathrm{C}_{2}$ repeats exhibit a nearly identical conformation except for the short turns connecting helix a and helix b (Fig. 3a). In addition, all dPPR repeats exhibit a high degree of structural homology with the repeats in RNA-bound PPR10 (PDB ID: 4M59) ${ }^{22}$, artificially engineered cPPR-polyC (PDB ID: 4WSL) ${ }^{29}$ and synthetic PPR protein synthPPR3.5 (PDB ID: 4OZS) ${ }^{35}$, indicating that our RNA-bound dPPR motifs fold similarly to the natural ones and to engineered proteins in their RNA-free form. In the dPPR- $\mathrm{U}_{8} \mathrm{C}_{2}$ structure, each helix $b$ stacks against the helix a of the following repeat through extensive van der Waals interactions, forming an interrepeat structure similar to a three-helix bundle (Fig. 3b). Furthermore, although the structures of cPPR-polyC, synthPPR3.5, RNA-bound PPR10 (repeat 6-15) and dPPR$\mathrm{U}_{8} \mathrm{C}_{2}$ all exhibit superhelical spiral shapes, they are distinct from one another in their configurational details. Although it has a similar diameter to RNA-free cPPR-polyC and synthPPR3.5, RNA-bound $\mathrm{dPPR}-\mathrm{U}_{8} \mathrm{C}_{2}$ exhibits a more compact conformation with a helical period length of $\sim 70 \AA$, which is shorter than that of cPPR proteins ( $\sim 90 \AA$; Fig. $3 \mathrm{c}$ ). The fact that the three types of engineered PPR motifs (dPPR, cPPR and synthPPR) are almost identical indicates that RNA binding may change the interaction between PPR hairpin-shaped motifs and induce conformational changes. Compared with RNA-bound PPR10 (repeats 6-15), RNA-bound dPPR- $\mathrm{U}_{8} \mathrm{C}_{2}$ also exhibits in a tighter form, and there is a 20 - $\AA$ difference between their diameters (Supplementary Fig. 5). According to previous studies, repeats 6-15 of PPR10 fail to bind RNA perfectly, thus suggesting that RNA binding might contribute to subtle conformational variations. We speculate that these differences may be gradually amplified over an increasing number of repeats, ultimately leading to the prominent compression of the superhelix. This conformational plasticity appears to be a result of extensive van der Waals interactions between adjacent repeats. A similar phenomenon has been observed in DNA-bound/unbound TALE crystal structures ${ }^{36,37}$ but not in the PUF-RNA interaction ${ }^{8,38}$.

Structural basis for specific RNA recognition by PPR code. As observed in all four complex structures, four types of dPPR repeats recognize their corresponding targets by forming hydrogen bonds with the Watson-Crick faces of the nucleotides, which explains why PPR proteins bind ssRNAs instead of double stranded ones. The electron densities of the RNA nucleotides 5 and 6 differ remarkably from each other (Supplementary Fig. 6), providing insight into the base-recognition mechanisms of PPR repeats. Previous studies have strongly suggested that the polar amino acid at the 5 th position in each repeat is the chief determinant of RNA base specificity. Serine or threonine at this position results in a preference for purines, whereas the presence of asparagine is correlated with a preference for pyrimidines. The 
a

b
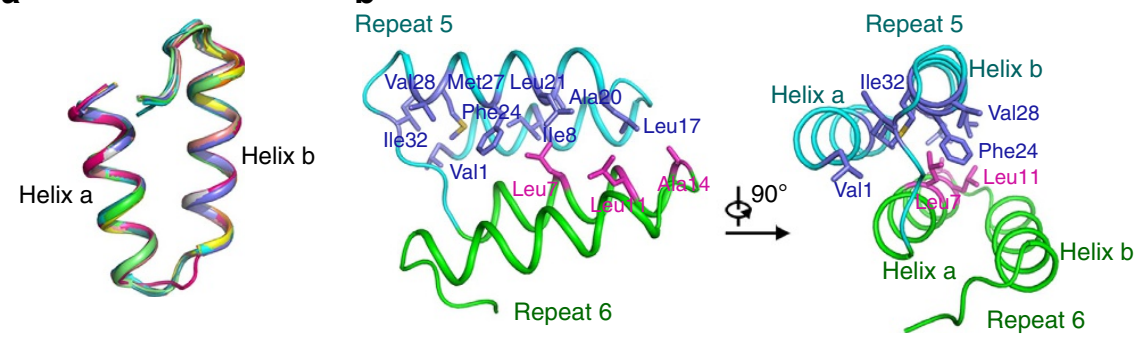

C
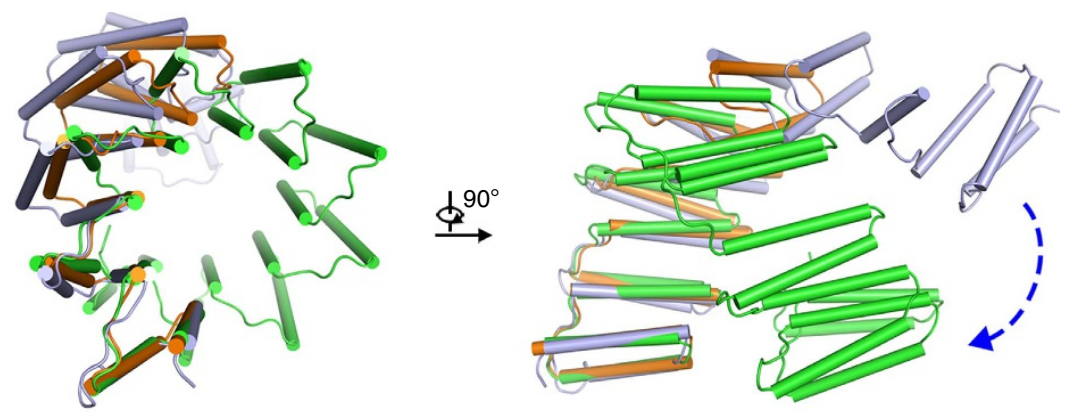

RNA-bound dPPR

synthPPR (PDB: 4OZS)

CPPR (PDB: 4WSL)

Figure 3 | Structural plasticity of $\mathbf{U}_{\mathbf{8}} \mathbf{C}_{\mathbf{2}}$. (a) All dPPR repeats of $d P P R-U_{8} C_{2}$ exhibit a nearly identical conformation. Each repeat is organized into two helices ( $a$ and $b$ ) followed by a short loop. (b) The contact between adjacent dPPR repeats is primarily mediated by van der Waals interactions. Repeats 5 and 6 from dPPR $-U_{8} C_{2}$ are coloured cyan and green, respectively. Two perpendicular views are presented, with amino acids that participate in the interaction shown in violet and magenta at repeats 5 and 6 , respectively. (c) RNA-bound dPPR- $U_{8} \mathrm{C}_{2}$ exhibits a more compressed conformation than RNAfree cPPR and synthPPR3.5. The three structures are superimposed using the first PPR repeat of each protein, and $d P P R-U_{8} C_{2}$, CPPR and synthPPR3.5 are coloured green, light blue and orange. The blue dashed arrow indicates the conformational differences between $d P P R-U_{8} C_{2}$ and cPPR.

structures of RNA-bound dPPRs provide a powerful explanation for these codes. The amide group of the Asn5 side chain donates a hydrogen bond to the $\mathrm{O} 2$ atom of the corresponding pyrimidine, whereas the $\mathrm{N} 3$ atom of purine accepts a hydrogen bond from the hydroxyl group of the corresponding amino acid (Fig. 4). The 35 th residue, which is the second significant amino acid of the PPR code, is also located in close proximity to the corresponding nucleobase. We observed that water molecules between bases and PPR repeats mediate hydrogen bonds between the polar residues and the bases in the cases of uracil and cytosine recognition. This recognition pattern has not been reported in the TALE-DNA ${ }^{36,37}$ or PUF-RNA interactions ${ }^{8,38}$. Each water molecule between the base and corresponding PPR repeat forms two hydrogen bonds: one with the $\mathrm{N} 3$ atom of the pyrimidine and one with the carboxyl group of Asp35 (Fig. 4a) or the hydroxyl group of Ser35 (Fig. 4b). Base selectivity is determined via 'water bridge' polarity. The N3 atom of uracil is a hydrogen bond donor, whereas the N3 atom of cytosine is a hydrogen bond acceptor. For purine, Asn35 or Asp35 form one (Fig. 4c) or two (Fig. 4d) hydrogen bonds with adenine and guanine, respectively. The $\mathrm{N} 1$ atom of adenine is a hydrogen bond acceptor, whereas both the N1 and N2 atoms of guanine are hydrogen bond donors. These structures demonstrate how the amino acids asparagine and aspartate at the 35 th position contribute to purine base selectivity.

Delineation of new PPR codes. In nature, the PPR code is degenerate ${ }^{15,25-27}$. Multiple combinations of amino acids at positions 5 and 35 can specify the same nucleotide, but sometimes the same combination of amino acids is similarly compatible with more than one type of nucleotide. Although we have not yet obtained crystal structures of PPR repeats with all code combinations, baserecognition models for codes such as $\mathrm{NN}, \mathrm{TN}$ and $\mathrm{SD}^{26,27}$ can be rationally deduced from the existing structural information (Fig. 5a). For instance, the code NN has been reported to be equally compatible with uracil and cytosine. Water-mediated hydrogen bonds may connect the amino acid at the 35th position with its coordinating pyrimidine. The ability of the side chain of Asn35 to rotate is also important because it allows the amino acid to be a hydrogen bond donor or acceptor. Under these circumstances, we predict that the N3 atom of either uracil or cytosine may form a hydrogen bond with the water molecule but with the opposite polarity. We designed and purified proteins containing predicted codes NN, TN and SD for biochemical verification (Fig. 5b). The biochemical results corroborated the models, demonstrating that the code NN exhibits similar selectivity towards $\mathrm{U}$ and $\mathrm{C}$ with dissociation constants of $\sim 15 \mathrm{nM}$, whereas codes TN and SD specifically recognize A and G, respectively (Fig. 5c). Thus, these results provide a framework for deciphering the RNA targets of the mysterious PPR-motif code, which comprises more than 30 code combinations in nature ${ }^{15,26,27}$.

\section{Discussion}

As a larger family of sequence-specific RNA-binding proteins, PPRs play various important roles in all aspects of organelles' RNA metabolism ${ }^{15}$. The dimerization states of PPR may function in the recognition of multiple RNA targets and the regulation of different signal responses. For example, PPR4 and PPR5 exist as monomers ${ }^{39,40}$, whereas HCF152 and PPR10 has been identified as homodimers $21,22,26,41$. Recently, two crystal structures of PPR in complex with RNA (PSAJ-bound PPR10 and YCF3-bound THA8) have revealed atypical modular and dimeric RNAtargeting modes 22,24 , whereas the solution structure of $A T P H$ PPR10 is monomeric ${ }^{30}$. In this study, each RNA-dPPR complex exhibited a monomeric and ideally modular RNA-binding mode, suggesting that the monomeric RNA-binding form of PPR is highly favourable under physiological conditions ${ }^{15}$. 
a

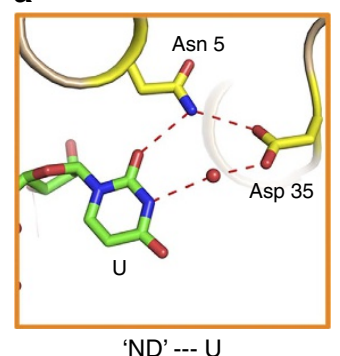

C

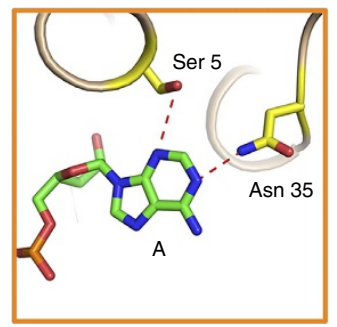

'SN' --- A b

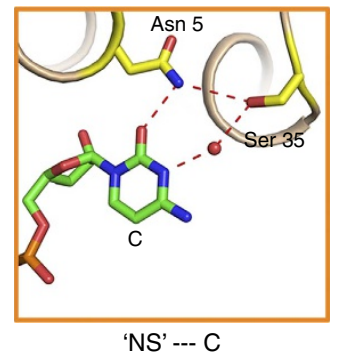

d

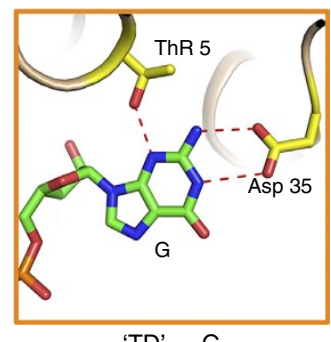

‘TD' --- G

Figure 4 | Structural basis of nucleobase recognition by dPPR repeats. PPR code amino acids selectively target nucleotides by forming direct or indirect hydrogen bonds to the Watson-Crick faces of bases. The specific recognition patterns of the bases $\mathrm{U}(\mathbf{a}), \mathrm{C}(\mathbf{b}), \mathrm{A}(\mathbf{c})$ and $\mathrm{G}(\mathbf{d})$ by dPPR repeats are shown in the zoom-in view. The side chains of the 5th and 35th residues in each PPR repeat are shown in yellow. Bases are labelled and coloured according to atom type (carbon: green, oxygen: red, nitrogen: blue). The hydrogen bonds are represented by red dotted lines. Water molecules are represented by red spheres. Single-letter abbreviations for the amino acid residues and nucleobases are as follows: D, Asp; N, Asn; S, Ser; T, Thr; A, Adenine; C, Cytosine; G, Guanine and U, Uracil.

Our data reveal that a dPPR protein recognizes its specific ssRNA target via hydrogen-bond-mediated interaction between the dPPR repeats and their coordinating nucleobases (Fig. 4). The most important feature reflected by our structures is that the amino acids at positions 5 and 35 in each repeat, which interact directly or indirectly with the target nucleobase, are critical for base selection. This finding provides structural evidence for PPR code theory, corroborating previous biochemical and computational results ${ }^{25-27}$. In addition, on the basis of our structures, the specific base recognition of PPR codes such as NN, TN and SD can be explained, thus providing a reference for bi-residue combinations. Given the dimension discrepancy between purine and pyrimidine, a PPR repeat that contains small amino acids such as alanine or glycine at position 5 can accommodate purine without notable steric clash but exhibits little specificity, potentially resulting in weak RNA-binding activity compared with 'TD' and 'SN'. Previous functional studies have focused on only a few PPR members because of the limitation imposed by RNA target identification. Herein, deciphering the codes for RNA recognition through use of dPPRs enabled the precise prediction of the RNA targets of numerous uncharacterized PPR proteins, and may provide a comprehensive understanding of the PPR family ${ }^{15,27}$.

Furthermore, our high-resolution structures provide precise information about RNA coordination by PPR repeats. Several additional important amino acids in dPPR repeats, such as Val2 and Lys13 (Supplementary Figs 7 and 8), also contribute to RNA binding. Together with its counterpart in the next repeat, each Val2 clamps its corresponding nucleobase in a sandwich-like manner through van der Waals interactions (Supplementary Fig. 7b), thus, explaining why the amino acids at this position are usually hydrophobic, as reported in a previous study ${ }^{2}$. Another

crucial amino acid involved in RNA binding is the lysine at position 13. Each phosphate group of the target ssRNA is oriented to helix a. Lys13 is positioned at the extremity of helix a in each repeat, contributing to the positive electrostatic potential facilitating interactions with the negatively charged phosphate (Supplementary Fig. 8a). Interactions with the phosphate group of ssRNA, which are invariant for repeats 1 through 8 , are mediated by salt bridges (Supplementary Fig. 8b) between Lys13 of repeat 1 and the $5^{\prime}$ phosphate group of U3, and between Lys13 of repeat 8 and the $5^{\prime}$ phosphate group of U10. Notably, Lys13 of repeat 9 also forms a salt bridge with the $3^{\prime}$ phosphate group of U10, but no interaction was observed between Lys13 of repeat 10 and ssRNA because of the poor electron density of the 3 '-terminal RNA nucleotides. The substitution of Lys13 with alanine in each repeat completely abolished RNA binding (Supplementary Fig. 8c), consistently with the results from a previous report ${ }^{29}$. These detailed analyses emphasize that the 33 other residues in addition to the two code residues must be considered when optimizing designed PPR repeats in future work.

Together, our structural analyses should help to improve the mechanistic perception of the PPR protein and facilitate the optimal design of useful tools for RNA manipulation with enhanced specificity and affinity. Furthermore, our work may serve as a model to explore the $\alpha$-helical repeat protein universe in silico ${ }^{42,43}$. Moreover, the detailed elucidation of the interaction mechanism between $\mathrm{dPPR}$ repeats and different nucleobases will allow the development of new types of dPPRs targeting modified nucleobases, including N6-methyladenosine ${ }^{44}$ and pseudouridine ${ }^{45-47}$, for potential biotechnological applications.

\footnotetext{
Methods

Protein preparation. All customized PPR genes were synthesized by Genewiz (GENEWIZ, Inc., China) and then subcloned into the pET21b vector (Novagen), resulting in recombinant $\mathrm{APPR}$ proteins fused with a $6 \times$ His tag at the $\mathrm{C}$-termin. The plasmids were transformed into E. coli BL21(DE3). One litre lysogeny broth medium supplemented with $100 \mu \mathrm{g} \mathrm{ml}^{-1}$ ampicillin was inoculated with a transformed bacterial pre-culture and shaken at $37^{\circ} \mathrm{C}$ until the optical density at $600 \mathrm{~nm}$ reached 1 . The culture was cooled to $16^{\circ} \mathrm{C}$ and induced with $0.2 \mathrm{mM}$ isopropyl- $\beta$-D-thiogalactoside. After growing for $16 \mathrm{~h}$ at $16^{\circ} \mathrm{C}$, the bacterial pellet was collected and homogenized in buffer A ( $25 \mathrm{mM}$ Tris- $\mathrm{HCl}, \mathrm{pH} 8.0,150 \mathrm{mM} \mathrm{NaCl})$. After sonication and centrifugation at $23,000 \mathrm{~g}$ at $4{ }^{\circ} \mathrm{C}$, the supernatant was loaded onto a column equipped with $\mathrm{Ni}^{2+}$ affinity resin (Ni-NTA, Qiagen), washed with buffer B ( $25 \mathrm{mM}$ Tris- $\mathrm{HCl}, \mathrm{pH} 8.0$, $150 \mathrm{mM} \mathrm{NaCl}, 15 \mathrm{mM}$ imidazole), and eluted with buffer C (25 mM Tris- $\mathrm{HCl}, \mathrm{pH}$ 8.0, $250 \mathrm{mM}$ imidazole) followed by ion exchange (Source 15Q, GE Healthcare). Each protein was then subjected to gel filtration chromatography (Superdex-200 10/300, GE Healthcare). The buffer for gel filtration contained $25 \mathrm{mM}$ Tris-HCl, pH 8.0, $100 \mathrm{mM}$ $\mathrm{NaCl}, 5 \mathrm{mM} \mathrm{MgCl}_{2}$ and $5 \mathrm{mM}$ 1,4-dithiothreitolDTT (Supplementary Fig. 9). The peak fractions were incubated with target RNA oligonucleotides at a molar ratio of $\sim 1: 1.5$ at $4{ }^{\circ} \mathrm{C}$ for $\sim 40 \mathrm{~min}$ before crystallization trials.
}

Crystallization. To obtain crystals of dPPR-RNA complexes, we first examined various combinations of $\mathrm{dPPR}-\mathrm{U}_{10}$ boundaries and corresponding RNA oligonucleotides (Takara). Finally, dPPR-U $\mathrm{U}_{10}$ (residues 123-572) and 18-nt RNA

$5^{\prime}$-ggggUUUUUUUUUUCccc- $3^{\prime}$ were crystallized in the reservoir solution $11-13 \%$ (w/v) polyethylene glycol 3,350, $100 \mathrm{mM}$ Bis-Tris propane, $\mathrm{pH} 6.5,150 \mathrm{mM} \mathrm{MgCl}_{2}$. However, the crystals exhibited poor diffraction, diffracting only to $8 \AA$. To generate dPPR- $U_{10}$ crystals with good X-ray diffraction, two rounds of additive screening were performed. The first additive screen revealed that ethyl acetate improved the diffraction to $3.5-4 \AA$. In this context, a second additive screen was then performed. Finally, the best crystals were obtained under the following conditions: $11-13 \%(\mathrm{w} / \mathrm{v})$ polyethylene glycol 3,350, Bis-Tris propane, $\mathrm{pH} 6.5,150 \mathrm{mM} \mathrm{MgCl}_{2}, 1.5 \%$ ethyl acetate and $3 \%(\mathrm{w} / \mathrm{v})$ $\mathrm{D}-(+)$-glucose monohydrate. The crystals were flash frozen in liquid nitrogen using a $2 \times$ mother solution as the cryoprotective buffer and diffracted beyond $2.3 \AA$ at Shanghai Synchrotron Radiation Facility (SSRF) beamline BL19U.

The other three proteins ( $\mathrm{dPPR}-\mathrm{U}_{8} \mathrm{C}_{2}, \mathrm{dPPR}-\mathrm{U}_{8} \mathrm{~A}_{2}$ and $\mathrm{dPPR}-\mathrm{U}_{8} \mathrm{G}_{2}$; residues 123-572) and their corresponding 18-ntRNAs with sequences of

$5^{\prime}$ ggggUUUUCCUUUU $\operatorname{cccc} 3^{\prime}$

$5^{\prime}$ ggggUUUUAAUUUU

$5^{\prime}$ ggggUUUUGGUUUUcccc $3^{\prime}$

yielded crystals in the same reservoir solution. All RNA-bound dPPR proteins were crystallized by the hanging-drop vapour-diffusion method at $18^{\circ} \mathrm{C}$ and mixed $1 \mu \mathrm{l}$ sample with an equal volume of reservoir solution. Crystals appeared overnight and grew to full size within 5-9 days. 
a

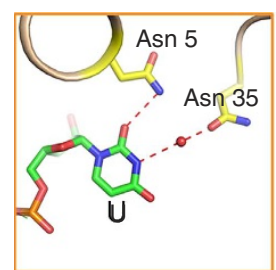

'NN' --- U

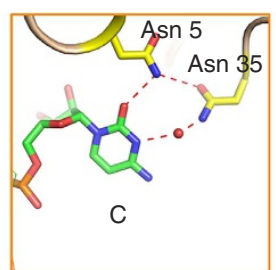

'NN' --- C

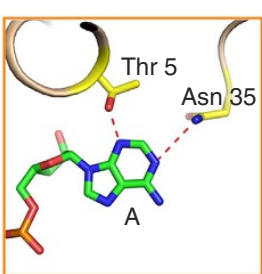

'TN' --- A

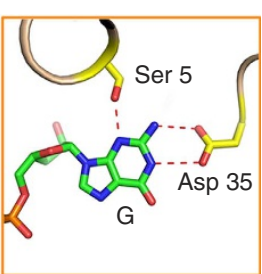

'SD' --- G

b

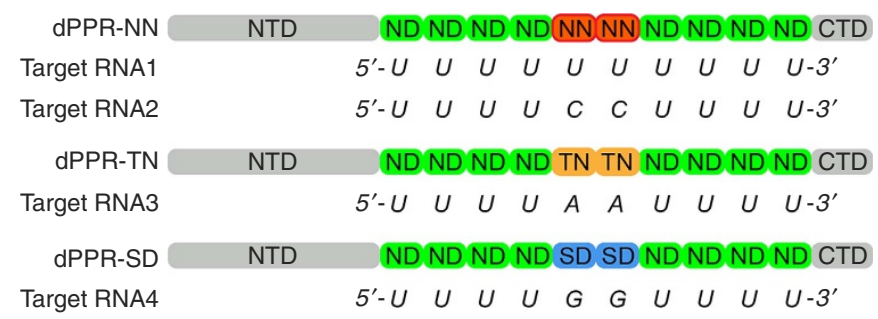

C
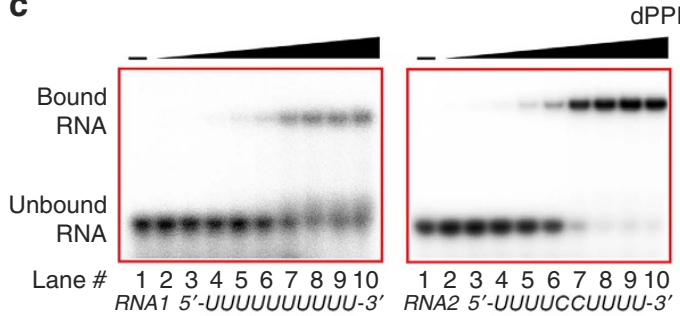

dPPR-NN

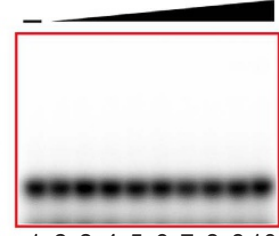

12345678910 RNA3 5'-UUUUAAUUUU-3
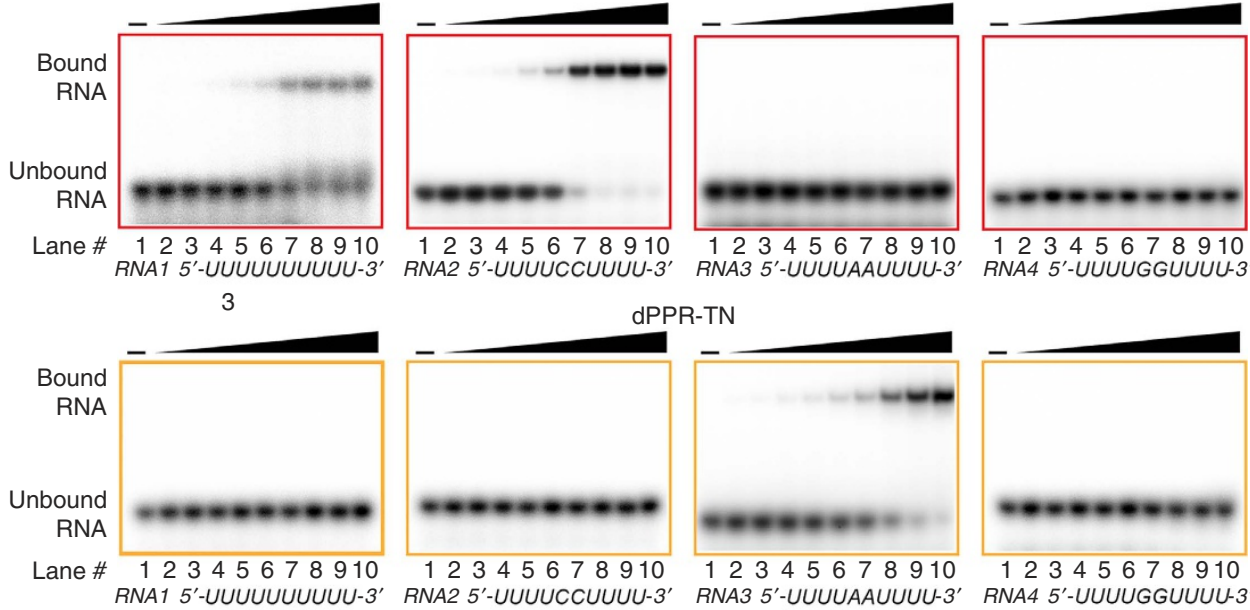

dPPR-SD
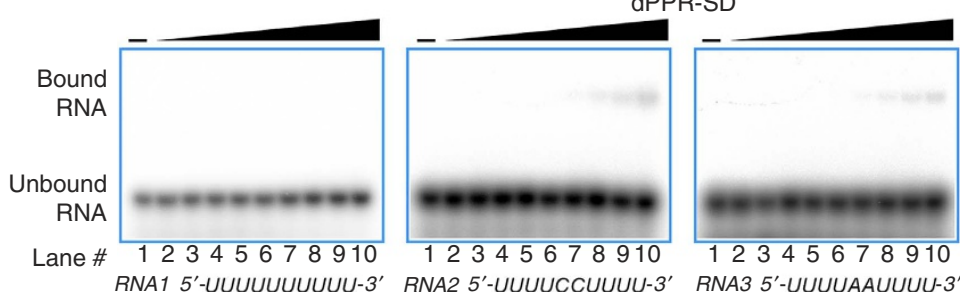

12345678910

RNA2 5'-UUUUCCUUUU-3'

RNA3 5'-UUUUAAUUUU-3'

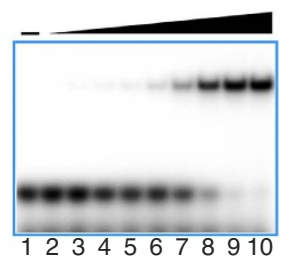

12345678910

Figure 5 I Interaction models for more PPR codes. (a) Prediction of another 4 interaction models for PPR codes 'NN', 'TN' and 'SD' with U, C, A and G, respectively. The hydrogen bonds are represented by red dotted lines. Water molecules are represented by red spheres. (b) Schematic representation of dPPR-NN, dPPR-TN, dPPR-SD and their target RNA sequences. The shaded binary amino acids are indicative of PPR repeats with different codes. NTD and CTD are from native PPR10. (c) EMSA demonstrates the specific RNA recognition of dPPR proteins with predicted codes. The final concentrations of dPPR in lanes 1-10 are 0, 0.8, 1.6, 3.2, 6.25, 12.5, 25, 50, 100 and $200 \mathrm{nM}$, respectively. The detailed $K_{d}$ values are shown in Supplementary Table 1.

Data collection and structural determination. All data sets were collected at SSRF beamline BL19U or BL17U and processed with the HKL3000 or HKL2000 packages $^{48}$. Further processing was performed with programs from the CCP4 suite ${ }^{49}$. Data collection and structure refinement statistics are summarized in Table 1 . The structure of the dPPR-RNA complex was solved by MR with the newly solved RNAfree structure as the search model using the programme PHASER ${ }^{50}$. The structure was manually iteratively refined with PHENIX and COOT $^{51,52}$ (Table 1).

Electrophoretic mobility shift assay (EMSA). The ssRNA oligonucleotides were radiolabelled at their $5^{\prime}$ ends with $\left[\gamma_{-}{ }^{32} \mathrm{P}\right]$ ATP (PerkinElmer), catalysed by T4 polynucleotide kinase (Takara). For EMSA, dPPR proteins were incubated with $\sim 2 \mathrm{nM}^{32} \mathrm{P}$-labelled probe in final binding reactions containing $25 \mathrm{mM}$ Tris- $\mathrm{HCl}$, $\mathrm{pH}$ 8.0, $5 \mathrm{mM} \mathrm{MgCl}_{2}, 5 \mathrm{mM}$ 1,4-dithiothreitol, $0.1 \mathrm{mg} \mathrm{ml}^{-1}$ bovine serum albumin, $50 \mathrm{ng} \mathrm{ml}^{-1}$ heparin and $10 \%$ glycerol for $20 \mathrm{~min}$ on ice. The reactions were then resolved on $8 \%$ native acrylamide gels (37.5: 1 acrylamide: bis-acrylamide) in $0.5 \times$ Tris-glycine buffer under an electric field of $15 \mathrm{~V} \mathrm{~cm}^{-1}$ for $40 \mathrm{~min}$. Gels were visualized on a phosphor screen (Amersham Biosciences) using a Typhoon Trio Imager (Amersham Biosciences). All presented images are representative of results from at least three independent experiments.

\section{References}

1. Hsu, P. D., Lander, E. S. \& Zhang, F. Development and applications of CRISPR-Cas9 for genome engineering. Cell 157, 1262-1278 (2014).

2. Filipovska, A. \& Rackham, O. Modular recognition of nucleic acids by PUF, TALE and PPR proteins. Mol. Biosyst. 8, 699-708 (2012).

3. Isalan, M. Zinc-finger nucleases: how to play two good hands. Nat. Methods. 9, 32-34 (2012). 
4. Bogdanove, A. J. \& Voytas, D. F. TAL effectors: customizable proteins for DNA targeting. Science 333, 1843-1846 (2011).

5. Bedell, V. M. et al. In vivo genome editing using a high-efficiency TALEN system. Nature 491, 114-U133 (2012).

6. Sternberg, S. H. \& Doudna, J. A. Expanding the Biologist's Toolkit with CRISPR-Cas9. Mol. Cell 58, 568-574 (2015).

7. Shalem, O., Sanjana, N. E. \& Zhang, F. High-throughput functional genomics using CRISPR-Cas9. Nat. Rev. Genet. 16, 299-311 (2015).

8. Wang, X. Q., McLachlan, J., Zamore, P. D. \& Hall, T. M. T. Modular recognition of RNA by a human Pumilio-homology domain. Cell 110, 501-512 (2002).

9. Ozawa, T., Natori, Y., Sato, M. \& Umezawa, Y. Imaging dynamics of endogenous mitochondrial RNA in single living cells. Nat. Methods 4, 413-419 (2007).

10. Campbell, Z. T., Valley, C. T. \& Wickens, M. A protein-RNA specificity code enables targeted activation of an endogenous human transcript. Nat. Struct. Mol. Biol. 21, 732-738 (2014).

11. Lurin, C. et al. Genome-wide analysis of Arabidopsis pentatricopeptide repeat proteins reveals their essential role in organelle biogenesis. Plant. Cell 16, 2089-2103 (2004).

12. Schmitz-Linneweber, C. \& Small, I. Pentatricopeptide repeat proteins: a socket set for organelle gene expression. Trends. Plant. Sci. 13, 663-670 (2008).

13. Yagi, Y., Nakamura, T. \& Small, I. The potential for manipulating RNA with pentatricopeptide repeat proteins. Plant J. 78, 772-782 (2014).

14. Manna, S. An overview of pentatricopeptide repeat proteins and their applications. Biochimie 113, 93-99 (2015).

15. Barkan, A. \& Small, I. Pentatricopeptide repeat proteins in plants. Annu. Rev. Plant. Biol. 65, 415-442 (2014).

16. Filipovska, A. \& Rackham, O. Pentatricopeptide repeats: modular blocks for building RNA-binding proteins. RNA Biol. 10, 1426-1432 (2013).

17. Small, I. D. \& Peeters, N. The PPR motif-a TPR-related motif prevalent in plant organellar proteins. Trends. Biochem. Sci. 25, 46-47 (2000).

18. Howard, M. J., Lim, W. H., Fierke, C. A. \& Koutmos, M. Mitochondrial ribonuclease $\mathrm{P}$ structure provides insight into the evolution of catalytic strategies for precursortRNA 5 ' processing. Proc. Natl Acad. Sci. USA 109, 16149-16154 (2012).

19. Ringel, R. et al. Structure of human mitochondrial RNA polymerase. Nature 478, 269-273 (2011).

20. Schwinghammer, K. et al. Structure of human mitochondrial RNA polymerase elongation complex. Nat. Struct. Mol. Biol. 20, 1298-1303 (2013).

21. Li, Q. et al. Examination of the dimerization states of the single-stranded RNA recognition protein pentatricopeptide repeat 10 (PPR10). J. Biol. Chem. 289, 31503-31512 (2014).

22. Yin, P. et al. Structural basis for the modular recognition of single-stranded RNA by PPR proteins. Nature 504, 168-171 (2013).

23. Ban, T. et al. Structure of a PLS-class pentatricopeptide repeat protein provides insights into mechanism of RNA recognition. J. Biol. Chem. 288, 31540-31548 (2013).

24. Ke, J. et al. Structural basis for RNA recognition by a dimeric PPR-protein complex. Nat. Struct. Mol. Biol. 20, 1377-1382 (2013).

25. Yagi, Y., Hayashi, S., Kobayashi, K., Hirayama, T. \& Nakamura, T. Elucidation of the RNA recognition code for pentatricopeptide repeat proteins involved in organelle RNA editing in plants. PLoS ONE 8, e57286 (2013).

26. Barkan, A. et al. A combinatorial amino acid code for RNA recognition by pentatricopeptide repeat proteins. PLoS Genet. 8, e1002910 (2012).

27. Cheng, S. et al. Redefining the structural motifs that determine RNA binding and RNA editing by pentatricopeptide repeat proteins in land plants. Plant J. 85, 532-547 (2016)

28. Shen, C. et al. Specific RNA recognition by designer pentatricopeptide repeat protein. Mol. Plant 8, 667-670 (2015).

29. Coquille, S. et al. An artificial PPR scaffold for programmable RNA recognition. Nat. Commun. 5, 5729 (2014).

30. Gully, B. S. et al. The solution structure of the pentatricopeptide repeat protein PPR10 upon binding atpH RNA. Nucleic Acids. Res. 43, 1918-1926 (2015).

31. Kotera, E., Tasaka, M. \& Shikanai, T. A pentatricopeptide repeat protein is essential for RNA editing in chloroplasts. Nature 433, 326-330 (2005).

32. Dahan, J. \& Mireau, H. The Rf and Rf-like PPR in higher plants, a fast-evolving subclass of PPR genes. RNA. Biol. 10, 1469-1476 (2013).

33. Khrouchtchova, A., Monde, R. A. \& Barkan, A. A short PPR protein required for the splicing of specific group II introns in angiosperm chloroplasts. RNA 18, 1197-1209 (2012)

34. Prikryl, J., Rojas, M., Schuster, G. \& Barkan, A. Mechanism of RNA stabilization and translational activation by a pentatricopeptide repeat protein. Proc. Natl Acad. Sci. USA 108, 415-420 (2011).

35. Gully, B. S. et al. The design and structural characterization of a synthetic pentatricopeptide repeat protein. Acta Crystallogr. D. Biol. Crystallogr. 71, 196-208 (2015).

36. Deng, D., Yan, C. Y., Wu, J. P., Pan, X. J. \& Yan, N. Revisiting the TALE repeat. Protein Cell 5, 297-306 (2014)

37. Deng, D. et al. Structural basis for sequence-specific recognition of DNA by TAL effectors. Science 335, 720-723 (2012).
38. Filipovska, A., Razif, M. F., Nygard, K. K. \& Rackham, O. A universal code for RNA recognition by PUF proteins. Nat. Chem. Biol. 7, 425-427 (2011).

39. Schmitz-Linneweber, C. et al. A pentatricopeptide repeat protein facilitates the trans-splicing of the maize chloroplast rps12 pre-mRNA. Plant Cell 18, 2650-2663 (2006).

40. Beick, S., Schmitz-Linneweber, C., Williams-Carrier, R., Jensen, B. \& Barkan, A The pentatricopeptide repeat protein PPR5 stabilizes a specific tRNA precursor in maize chloroplasts. Mol. Cell Biol. 28, 5337-5347 (2008).

41. Meierhoff, K., Felder, S., Nakamura, T., Bechtold, N. \& Schuster, G. HCF152, an Arabidopsis RNA binding pentatricopeptide repeat protein involved in the processing of chloroplast psbB-psbT-psbH-petB-petD RNAs. Plant Cell 15, 1480-1495 (2003).

42. Doyle, L. et al. Rational design of alpha-helical tandem repeat proteins with closed architectures. Nature 528, 585-588 (2015).

43. Brunette, T. J. et al. Exploring the repeat protein universe through computational protein design. Nature 528, 580-584 (2015).

44. Yue, Y., Liu, J. \& He, C. RNA N6-methyladenosine methylation in posttranscriptional gene expression regulation. Genes Dev. 29, 1343-1355 (2015).

45. Schwartz, S. et al. Transcriptome-wide mapping reveals widespread dynamicregulated pseudouridylation of ncRNA and mRNA. Cell 159, 148-162 (2014).

46. Carlile, T. M. et al. Pseudouridine profiling reveals regulated mRNA pseudouridylation in yeast and human cells. Nature 515, 143-146 (2014).

47. Li, X. et al. Chemical pulldown reveals dynamic pseudouridylation of the mammalian transcriptome. Nat. Chem. Biol. 11, 592-597 (2015).

48. Otwinowski, Z., Minor, W. \& W, Jr C. C. Processing of X-ray diffraction data collected in oscillation mode. Macromolecular Crystallography Part A 276, 307-326 (1997).

49. Collaborative Computational Project, Number4. The CCP4 suite: programs for protein crystallography. Acta Crystallogr. D. 50, 760-763 (1994).

50. Mccoy, A. J. et al. Phaser crystallographic software. J. Appl. Crystallogr. 40, 658-674 (2007).

51. Adams, P. D. et al. PHENIX: building new software for automated crystallographic structure determination. Acta Crystallogr. D 58, 1948-1954 (2002).

52. Emsley, P. \& Cowtan, K. Coot: model-building tools for molecular graphics. Acta Crystallogr. D: Biol. Crystallogr. 60, 2126-2132 (2004).

\section{Acknowledgements}

Authors would like to thank J. He at Shanghai Synchrotron Radiation Facility (SSRF) beamline BL17U and R. Zhang at SSRF beamline BL19U for on-site assistance and Professor J.W. Wang at the College of Life Science, Tsinghua University, for data collection and technical support. Authors also thank research associates at Center for Protein Research (CPR) and State Key Laboratory of Agricultural Microbiology, Huazhong Agricultural University, for technical support. This work was supported by funds from the Ministry of Science and Technology (Grant Number 2015CB910900), the Fundamental Research Funds for the Central Universities (Program No. 2014PY026, No. 2015PY219 and No. 2014JQ001) and Huazhong Agricultural University Scientific \& Technological Self-innovation Foundation (Program No. 2013RC013).

\section{Author contributions}

C.S., T.Z., D.Z., Y.L. and P.Y. designed all experiments. C.S., D.Z., Z.G., Y.L., Z.Y., Y.Y., X.W., Q.W., Q.Z., S.F., T.Z. and P.Y. performed the experiments. All authors analyzed the data and contributed to manuscript preparation. C.S., T.Z., Y.L. and P.Y. wrote the manuscript.

\section{Additional information}

Accession Codes: The atomic coordinates and structure factors for the four structures in complex with RNA have been deposited in the Protein Data Bank (PDB) with the accession codes 5I9F (poly- $\mathrm{U}_{10}$ ), 5I9G (poly- $\mathrm{U}_{8} \mathrm{C}_{2}$ ), 5I9D (poly- $\mathrm{U}_{8} \mathrm{~A}_{2}$ ) and $5 \mathrm{I} 9 \mathrm{H}\left(\right.$ poly- $\mathrm{U}_{8} \mathrm{G}_{2}$ ).

Supplementary Information accompanies this paper at http://www.nature.com/ naturecommunications

Competing financial interests: The authors declare no competing financial interests

Reprints and permission information is available online at http://npg.nature.com/ reprintsandpermissions/

How to cite this article: Shen, C. et al. Structural basis for specific single-stranded RNA recognition by designer pentatricopeptide repeat proteins. Nat. Commun. 7:11285 doi: $10.1038 /$ ncomms11285 (2016).

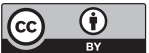

This work is licensed under a Creative Commons Attribution 4.0 International License. The images or other third party material in this article are included in the article's Creative Commons license, unless indicated otherwise in the credit line; if the material is not included under the Creative Commons license, users will need to obtain permission from the license holder to reproduce the material. To view a copy of this license, visit http://creativecommons.org/licenses/by/4.0/ 